\title{
Comparación de un programa de ejercicio intradiálisis frente a ejercicio domiciliario sobre capacidad física funcional y nivel de actividad física
}

\author{
Lucía Ortega Pérez de Villar, Sara Antolí García, Ma Jesús Lidón Pérez, Juan José Amer Cuenca, Vicent \\ Benavent Caballer, Eva Segura Ortí
}

Departamento fisioterapia Universidad CEU Cardenal Herrera. Hospital Universitario Doctor Peset. Valencia. España

\begin{abstract}
Resumen
Introducción: El ejercicio durante la hemodiálisis es beneficioso, aunque son pocas las unidades de hemodiálisis que ofrecen un programa de ejercicio adaptado a estos pacientes. Por ello es necesario encontrar alternativas más económicas para realizar ejercicio. El objetivo es comparar los efectos de un programa de ejercicio intradiálisis frente a ejercicio domiciliario, sobre la adherencia al programa, la capacidad física funcional y el nivel de actividad física.
\end{abstract}

Métodos: 17 pacientes en hemodiálisis de un centro de Valencia fueron aleatorizados dividiéndoles en un grupo de ejercicio intradiálisis $(n=9)$ y un grupo domiciliario $(n=8)$. Ambos programas incluían ejercicio aeróbico y de fuerza durante 4 meses. Se valoró una amplia batería de pruebas funcionales (Short Physical Performance Battery, equilibrio monopodal, Timed Up and Go, Sit To Stand to sit test 10 y 60, dinamometría de mano, fuerza de tríceps, 6 minutos marcha) y dos cuestionarios de nivel de actividad física (Human Activity Profile y Physical Activity Scale for Elderly).

Resultados: 2 pacientes del grupo intradiálisis y 5 pacientes de ejercicio domiciliario finalizaron el programa y fueron analizados. Se observó un efecto significativo del factor tiempo en el caso del Human Activity Profile $(P<.017)$. En las pruebas funcionales no se encontró ninguna diferencia significativa. En cuanto a la adherencia al ejercicio los pacientes del grupo intradiálisis cumplieron el $92.7 \%$ y el grupo domiciliario el $68.7 \%$ del total de las sesiones.

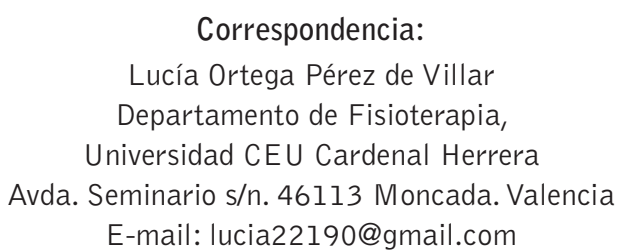

Conclusiones: En ambos grupos se observa un aumento del nivel de actividad física. Sin embargo, es necesario modificar factores, tanto en el personal sanitario como en los propios pacientes, para conseguir mayor adherencia a los programas de ejercicio.

PALABRAS CLAVE

- EJERCICIO INTRADIÁLISIS

- HOME BASED

- INSUFICIENCIA RENAL CRÓNICA

- ACTIVIDAD FÍSICA

Comparison of intradialysis exercise program versus home exercise on functional capacity and physical activity level

\section{Abstract}

Introduction: Although exercise training of patients undergoing hemodialysis is generally associated with positive outcomes, few hemodialysis units routinely offer intradialytic exercise therapy. This is often related to financial cost and/or staff limitations. Home-based. The aim of this study was to compare the effects of intradialytic versus home based exercise regarding adherence, functional capacity and physical activity level.

Methods: 17 participants from hemodialysis unit from Valencia were randomized to either intradialytic exercise $(n=9$,$) or home based exercise (n=8)$. Both programs consisted of a combination of strength training and aerobic exercise during 4 months. We assess functional capacity with different test (Short Physical Performance Battery, one leg stand, Timed Up and Go, Sit To Stand test 10 and 60,handgrip, one leg heel rise, 6 minutes walking test) and two questionnaires to asses physical activity level (Human Activity Profile and Physical Activity Scale for Elderly). 
Outcomes: 2 participant from the intradialysis exercise and 5 participants from de home based exercise were analysed. We observed a significant time effect in the Human Activity Profile $(P<.017)$. In the functional capacity test there was not a significant difference. The subjects from the intradilysis exercise completed the $92.7 \%$ and the home based group the $68.7 \%$ of the total of the sessions.

Conclusions: In both groups was an increment of the physical activity. Nevertheless, it is necessary to modify some factors, both in health professionals and patients, to achieve higher.

\section{KEYWORDS}

- INTRADIALYSIS EXERCISES

- HOME BASED

- CHRONIC KIDNEY DISEASE

- PHYSICAL ACTIVITY

\section{Introducción}

Los pacientes con Insuficiencia Renal Crónica (IRC) en estadio terminal, pueden ser sometidos a hemodiálisis (HD) como tratamiento renal sustitutivo, el cual suple las principales funciones del riñón.

A medida que van pasando los años, la HD va afectando a los diferentes sistemas del organismo, entre los que destacan el sistema cardiovascular ${ }^{1,2}$ y el músculo-esquelético ${ }^{1,3,4,5}$, aunque estos pacientes también sufren alteraciones psicosociales. Es común encontrar en los pacientes en HD un bajo nivel de calidad de vida relacionado con la salud, asociado frecuentemente a la presencia de ansiedad y depresión ${ }^{6,7,8,9}$.

Esto no es solo debido a la HD. Estos pacientes tienen un nivel de actividad significativamente inferior al que presentan sus homólogos sanos ${ }^{10}$. De hecho hay estudios que demuestran que la mayoría de los pacientes que están en este tipo de tratamiento tienen una vida sedentaria ${ }^{11}$. Es por ello, que esta población presenta una disminución de la capacidad funcional ${ }^{12,13}$ que provoca una dependencia en las actividades de la vida diaria, discapacidad, así como un incremento del riesgo de hospitalización y mortalidad $^{14}$.

El ejercicio físico se viene utilizando como herramienta terapéutica en estos pacientes desde principios de los años $80^{15}$. Hay tres tipos de modalidades para los programas de ejercicio terapéutico: ejercicio en casa, ejercicio supervisado en días de no diálisis o ejercicio durante la sesión de HD. En el ejercicio intradiálisis es donde mejores resultados se ha obtenido por el control de constantes del paciente durante el ejercicio y por ser la modalidad que más sesiones de seguimiento consigue $^{16}$. La literatura dice que haciendo una combinación de ejercicio aeróbico y de resistencia muscular los pacientes obtienen mejores resultados ${ }^{17}$.Sin embargo, a día de hoy, son muy pocas las unidades que tienen implantado el ejercicio como una rutina durante las sesiones de $H D^{18}$, y una posible causa puede ser el coste económico que esto supone. Es por ello, que la implantación de un programa de ejercicio domiciliario podría suponer una posibilidad menos costosa para intentar conseguir mejorar la capacidad funcional de estos pacientes.

Por tanto, el objetivo principal de este estudio fue comparar los efectos de un programa de ejercicio intradiálisis frente a ejercicio domiciliario, sobre la adherencia al programa, la capacidad física funcional y el nivel de actividad física.

\section{Material y métodos}

\section{Sujetos}

Se incluyeron a diecisiete pacientes con insuficiencia renal crónica en estadio terminal de la unidad de diálisis al Hospital Virgen del Consuelo de Valencia (España) para participar en el estudio entre octubre de 2014 y enero de 2015. El estudio fue aprobado por el Comité de Ética y los participantes firmaron el consentimiento informado. La investigación se realizó dentro de las pautas marcadas por la Declaración de Helsinki de 1975.

Los criterios de inclusión fueron: estar al menos 3 meses en tratamiento de hemodiálisis y encontrarse estable médicamente. Los criterios de exclusión fueron: (1) Infarto de miocardio en las 6 semanas previas (2) angina inestable al ejercicio o en reposo (3) amputación de miembros inferiores por encima de rodilla sin prótesis (4) enfermedad vascular cerebral (ictus, isquemias transitorias) (5) alteraciones músculo-esqueléticas 0 respiratorias que empeoren con el ejercicio (6) imposibilidad de realizar las pruebas funcionales.

Se dividieron a los sujetos en dos grupos de manera aleatorizada: un grupo de ejercicio intradiálisis (GI) $(n=9)$ y otro grupo de ejercicio domiciliario (GD) $(n=8)$ que voluntariamente quisieron realizar el ejercicio. 
Se tomaron los siguientes datos clínicos al inicio del estudio: edad, peso seco, índice de masa corporal, tiempo en HD, horas de HD a la semana.

La capacidad física funcional y el nivel de actividad física se midieron al inicio y al final del programa.

\section{Capacidad física funcional}

Las pruebas funcionales encaminadas a evaluar la condición física de los pacientes fueron el Short Physical Performance Battery (SPPB), el equilibrio monopodal, el Timed up and go (TUG), el Sit to stand to sit test 10 y 60 (STS 10 y STS 60), la dinamometría de mano, la fuerza de tríceps sural, y el 6 minutos marcha (6MWT).

La prueba Short Physical Performance Battery (SPPB) se registró el primer día antes de la sesión de HD. Consta de tres pruebas de equilibrio, con los pies juntos, en posición de semitándem y en tándem, y se valora si el sujeto puede mantener cada una de estas posiciones hasta un máximo de 10 segundos. La valoración del equilibrio va de 0 a 4 puntos. Incluye también una prueba de velocidad de la marcha en 4 metros a velocidad habitual. Se realizaron dos intentos cronometrados y se registró el mejor de ellos, de forma que, según los segundos empleados, se asignó una puntuación de 1 a 4 . Se valoró también la prueba STS-5, que consiste en medir el tiempo empleado en levantarse y sentarse de la silla 5 veces con los brazos cruzados en el pecho, deteniendo el cronómetro cuando el paciente alcanzaba la posición de pie en la repetición 5. Según el tiempo empleado se asignó una puntuación de 0 a 4 puntos. Por lo tanto, la puntuación del SPPB va de 1 a 12 puntos $^{19}$.

Se valoró también el equilibrio estático monopodal antes de la primera sesión semanal de HD, pidiendo a los sujetos que levantasen una pierna flexionándola de forma que se pusieses a la pata coja, con la pierna que se encontrasen más seguros. Se les pidió que aguantasen el equilibrio el mayor tiempo posible. El fisioterapeuta anotó el tiempo que el paciente aguantaba en posición de apoyo monopodal. El sujeto podía mover los brazos y flexionar la rodilla si lo necesitaba para mantener el equilibrio. El tiempo finalizaba cuando el sujeto usaba sus brazos para apoyarse, utilizaba el pie elevado para apoyarse en el suelo, cuando el pie apoyado se movía para mantener el equilibrio o cuando el tiempo llegaba a los 45 segundos. Se repitió el procedimiento 3 veces y se registró el mejor tiempo ${ }^{20}$.

La prueba "Timed Up and Go" (TUG) 21,22, también se realizó previamente a la primera sesión de hemodiálisis.
Desde una silla el paciente se tenía que levantar, caminar 3 metros y volver a la posición inicial. Se repitió el procedimiento 3 veces y se registró el mejor tiempo.

La prueba de dinamometría de mano se realizó con el Dinamómetro JAMAR previamente al segundo día de la sesión semanal de HD. Según varios estudios en pacientes en diálisis, existe una correlación entre la fuerza de prensión de la mano con su estado de salud23,24. Los sujetos sentaban en una silla, con los pies apoyados en el suelo y las rodillas flexionadas $90^{\circ}$. El hombro del brazo que se estaba registrando permanecía en rotación neutra, con el codo en flexión de $90^{\circ}$ apoyado en la mesa, muñeca y antebrazo semipronados $\left(0-30^{\circ}\right)$ y una desviación cubital entre 0 y $15^{\circ}$. Se realizaron 3 repeticiones consecutivas, con un descanso de 15 segundos entre ellas, en cada uno de los miembros superiores, comenzando por el brazo dominante. Se dio ánimo verbal en las repeticiones y se registró la de valor máximo.

El segundo día de la sesión semanal de hemodiálisis también se realizaron las pruebas del STS 10 y STS 60, tal y como describen Cüska y McCarty25. La primera consiste en medir los segundos que necesita el paciente para levantarse y volver a sentarse 10 veces consecutivas lo más rápido posible, desde una posición de sentado y con los brazos cruzados en el pecho. Tras realizar las 10 repeticiones se registró el tiempo que había necesitado para realizar la prueba, así como el grado de dificultad en la Escala de Esfuerzo Percibido (EEP) (Figura 1).

\section{ESCALA DE ESFUERZO PERCIBIDO (EEP)}

¿Cómo nota el ejercicio?

\begin{tabular}{|l|l|}
\hline 6 & - \\
\hline 7 & Muy, muy suave \\
\hline 8 & - \\
\hline 9 & Muy suave \\
\hline 10 & - \\
\hline 11 & Ligero \\
\hline 12 & - \\
\hline 13 & Algo duro \\
\hline 14 & - \\
\hline 15 & Duro \\
\hline 16 & - \\
\hline 17 & Muy duro \\
\hline 18 & - \\
\hline 19 & Muy, muy duro \\
\hline 20 & Máximo esfuerzo percibido \\
\hline
\end{tabular}

Figura 1. Escala de Esfuerzo Percibido (EEP). 
A continuación, tras un periodo de recuperación de 3 minutos como mínimo, se realizó el STS 60 . Se explicó al paciente que la prueba consistía en realizar el máximo número de repeticiones de levantarse y volver a sentarse en un tiempo de 60 segundos. Tras la realización de la prueba se registraron las repeticiones y se anotó el grado de dificultad de la prueba según la EEP.

La prueba de la fuerza del tríceps sural o prueba de elevación de talón monopodal, se realizó también inmediatamente antes de la segunda sesión semanal de $H^{26,27,28}$. Los pacientes debían de estar descalzos. El ritmo de elevación del talón se marcó mediante un metrónomo. Antes de iniciar la prueba se pidió al paciente que mantuviese el equilibrio sobre una sola pierna, con un apoyo ligero de las puntas de los dedos en la pared, con los brazos separados del tronco y evitando que trasladasen el peso a través de los brazos a la pared, mientras el pie contralateral se situó ligeramente elevado del suelo. Se permitió un intento de elevación con el pie izquierdo, tras el cual se procedió a valorar la elevación del talón derecho. Se les pidió que elevaran el talón todo lo que pudieses al ritmo del metrónomo hasta que no pudieses elevar el talón por fatiga muscular. Si el sujeto compensaba con los brazos contra la pared o flexionaba la rodilla terminaba la prueba. También finalizaba si alcanzaba las 25 repeticiones, pues se ha establecido que estas son las repeticiones que de media se encuentran en la población sana ${ }^{26,27}$. Se anotó el número de repeticiones por cada pierna así como el grado de dificultad según la EEP.

La prueba de 6 minutos marcha (6MWT) se realizó previamente a la tercera sesión semanal de hemodiálisis, en el pasillo de la unidad. En el momento previo a la realización de la prueba se registró, mediante un esfigmomanómetro digital, la tensión arterial y la frecuencia cardiaca en el brazo sin fístula. A continuación se le indicó al paciente que durante 6 minutos, debía recorrer la máxima distancia señalada por unas marcas en el suelo, girando sin parar cada vez que recorría 20 metros. La orden dada al paciente fue la siguiente: «tiene que intentar recorrer la máxima distancia posible en este pasillo de 20 metros, andando lo más rápido que pueda sin llegar a correr». Se permitió realizar el test con ayudas para la deambulación si el paciente las utilizaba en su vida diaria o con la asistencia de otra persona, o parar y reiniciar la marcha en caso de necesitar un descanso durante el test. Se registró la distancia recorrida y se pidió al paciente que describiera en la EEP cuál había sido el grado de dificultad de la prueba ${ }^{29}$.

\section{Nivel de actividad física}

La valoración del nivel de actividad física de los sujetos se realizó mediante dos cuestionarios en la versión en español, el Physical Activity Scale for the Elderly (PASE) y el cuestionario Human Activity Profile (HAP). Ambos cuestionarios han sido validados en la población con enfermedad renal ${ }^{8}$. EI PASE fue diseñado para valorar la actividad realizada la semana previa según la respuesta de los sujetos a una serie de cuestiones sobre el tiempo empleado en actividades cotidianas. El total de la puntuación se calcula como la suma de tiempo en cada actividad multiplicado por la carga de la actividad ${ }^{8}$.

EI HAP es un cuestionario de 94 ítems, que valora la participación en actividades que requieren diferente cantidad de aporte energético. Los sujetos deben asignar cada actividad en una de las categorías: (1) puedo realizar esta actividad, (2) he dejado de realizar esta actividad) y (3) nunca he realizado esta actividad. Se valora tanto el máximo nivel de actividad como la puntuación de actividad ajustada, que se considera un estimador más estable de la actividad cotidiana del sujeto ${ }^{30}$. La puntuación de actividad máxima (MAS) es el número de ítems clasificados como el máximo consumo de oxígeno requerido que el sujeto puede realizar. La puntuación de actividad ajustada (AAS) es el resultado de restar al MAS y el número de actividades que el sujeto ha dejado de realizar, dando una mejor estimación del rango de actividades que puede realizar y la presencia de incapacidad. Por ejemplo, un sujeto cuya actividad más vigorosa que puede seguir realizando es la de "subir 36 peldaños" (ítem número 60 ) tendrá un MAS de 60. Si el individuo ya no realiza 6 actividades que requieren menos gasto energético que subir 36 peldaños de una escalera, su AAS será 54. Así pues, la puntuación del HAP representa un rango de actividades que una persona puede realizar en lugar de la actividad real realizada durante un periodo de tiempo determinado. EI HAP ha mostrado ser fiable para valorar la actividad física en sujetos en $\mathrm{HD}^{8,31}$.

\section{Intervención de ejercicio de fuerza-resistencia intradiálisis}

La intervención tuvo una duración de 4 meses, y fue realizada durante las 2 primeras horas de hemodiálisis, los tres días semanales que el paciente acudió a tratamiento. El programa de ejercicio consistió en la realización de ejercicios isotónicos e isométricos de resistencia progresiva con objeto de potenciar la musculatura de los miembros inferiores. Cada sesión de ejercicio se estructuró en un calentamiento, una parte principal y una vuelta a la calma. La sesión de ejercicio comenzaba y terminaba con unos 5 minutos de ejercicios de estiramientos de tríceps sural, isquiotibiales y movilizaciones 
de cadera. La parte principal de trabajo consistía en una serie de ejercicios de potenciación muscular, adaptado a la posición en que el paciente realizaba la HD. El primer ejercicio consistía en una extensión de la rodilla desde $\operatorname{los} 90^{\circ}$ a los $0^{\circ}$ con lastres en el tobillo, con un ritmo de contracción controlado mediante un metrónomo digital y vuelta a la posición inicial (2 segundos de contracción concéntrica 2 segundos de contracción isométrica y cuatro segundos de contracción isotónica excéntrica, sin pausa entre repeticiones). La carga se determinó con la prueba de 10 RM (repetición máxima) y de forma que percibiera un esfuerzo de entre 12 y 15 EEP.

El segundo ejercicio consistía en realizar una dorsiflexión y plantiflexion de tobillo con goma elástica. La banda elástica se colocaba a nivel de las cabezas de los metatarsianos. El ritmo de contracción estaba controlado por un metrónomo digital y consistía en realizar contracción excéntrica de 1 segundo (dorsiflexión) y contracción concéntrica de 2 segundos (plantiflexión). Se aumentaba la resistencia poniendo otra banda elástica. El paciente tenía que realizar 15 repeticiones.

El tercer ejercicio consistió en realizar una triple extensión de tobillo, rodilla y cadera contra una banda elásti$\mathrm{ca}$, de forma que se estandarizaba la posición de partida en $90^{\circ}$ de flexión de cadera, rodilla y tobillo. La banda elástica se colocaba a nivel de las cabezas metatarsianas y se pedía una triple extensión de 2 segundos de contracción concéntrica y 2 segundos de contracción excéntrica. La progresión en resistencia se consigue colocando una segunda banda elástica.

El cuarto ejercicio consistía en realizar isométricos de los aductores colocando una pelota entre las rodillas del pacientes Se les pedía que hicieran fuerza con las piernas, apretando el balón, a la vez que apretaban también los glúteos. El paciente tenía que aguantar cada contracción durante 6 segundos en tiempo espiratorio. El paciente realizó 15 repeticiones en cada una de las sesiones de ejercicio.

El quinto ejercicio consistió en un isométrico de isquiotibiales y el paciente se tenía que colocar la pelota entre el bajo del sillón y detrás de los talones. Se les pedía que hicieran fuerza con los talones hacia atrás en tiempo espiratorio. Tenían que aguantar la contracción durante 6 segundos. El paciente realizaba 15 repeticiones en cada una de las sesiones.

Durante todos los ejercicios se aseguró que la respiración fuera correcta, realizando los esfuerzos en espiración y evitando la maniobra de Valsalva.
Una vez finalizados los ejercicios de fuerza el paciente tenía que realizar bicicleta. La resistencia de la bicicleta se subía hasta que el paciente percibiera un esfuerzo de entre 12 y 15 EEP. El tiempo de la bicicleta se iba incrementando progresivamente durante el programa. Así es que el paciente comenzó pedaleando 15 minutos y terminó pedaleando 45 minutos.

Se realizó un seguimiento de los días que los participantes realizaban el ejercicio con un diario.

\section{Intervención de ejercicio de fuerza-resistencia domiciliaria}

El paciente tenía que realizar ejercicio en casa 3 días semana los días que a él le vinieran bien, ya fueran días de diálisis como días de no diálisis. Al comienzo, y durante tres sesiones, un fisioterapeuta le dio indicaciones sobre intensidad (se tenían que monitorizar la frecuencia cardiaca, la tensión arterial y siguiendo la EEP), frecuencia (mínimo 3 veces por semana) y modalidad de ejercicio (combinación de fuerza y aeróbico) que tenían que realizar. Se les entregó un dossier donde tenían toda la información necesaria para que realizaran el ejercicio en casa de forma independiente (detalle de los ejercicios con fotografías, repeticiones y series), así como un diario de registro de actividad.

Los ejercicios de los que constaba este programa eran similares a los realizados por el grupo intradiálisis. Se realizó un seguimiento por parte del fisioterapeuta cada vez que iba a la unidad de diálisis a realizar el ejercicio durante la diálisis, de modo que durante los dos primeros meses les preguntaba una vez por semana y posteriormente 1 vez al semana. Si los participantes tenían cualquier duda se podían poner en contacto con el fisioterapeuta, para aclarar dudas o resolver cualquier problema que existiera con el programa, tanto por contacto telefónico como en la misma unidad de diálisis.

\section{Análisis estadístico}

Dado el tamaño reducido de la muestra, los datos obtenidos no se ajustan a una distribución normal (test de Kolmogorov Smirnov, asimetría y curtosis). Se utilizó la prueba no paramétrica $U$ de Mann-Whitney para comprobar si había diferencia entre los grupos en características iniciales de los grupos. Se analizó el efecto de los programas de ejercicio mediante un test ANOVA mixto, con el factor tiempo intra-grupo y el grupo de intervención como factor entre grupos. Los datos se presentan 
como mediana, mínimo y máximo, o como media y desviación estándar. El análisis estadístico se realizó mediante el programa SPSS 20.0 para Windows (SPSS Inc., Chicago, III) tomando como significativos valores de $p<0.05$.

\section{Resultados}

En el GI había 4 hombres y 5 mujeres, mientras que en el GD estaba formado por 4 hombres y 4 mujeres.

Del GI se produjeron 7 bajas a lo largo del estudio: una persona le tocó el GI y quería realizar ejercicio a domicilio; dos personas abandonaron por problemas cardíacos y/o de tensión arterial durante el ejercicio; una persona abandonó por problemas articulares; una persona abandonó por fractura de tibia; una persona abandonó por ingreso prolongado en el hospital; una persona falleció. En el GD se produjeron 3 bajas: una persona abandonó porque no quería realizar ejercicio a domicilio, otra persona abandonó por fractura de tibia y otra persona fue trasplantada.

La tabla 1 muestra los datos de la historia clínica de los pacientes. Se puede observar que no hay diferencias significativas entre los grupos en términos de edad o índice de masa corporal.

Tabla 1. Datos historia clínica.

\begin{tabular}{|l|l|l|l|}
\hline Variable & $\begin{array}{l}\text { Grupo Intradiálisis } \\
n=2 \\
\text { X (DE) }\end{array}$ & $\begin{array}{l}\text { Grupo Domiciliario } \\
n=5 \\
\mathbf{X}(\mathbf{D E})\end{array}$ & $\mathbf{P}$ \\
\hline $\begin{array}{l}\text { Edad } \\
\begin{array}{l}\text { Índice de Masa Corporal } \\
\left(\mathrm{kg} / \mathrm{m}^{2}\right)\end{array}\end{array}$ & $\begin{array}{l}67(7.649) \\
(9.192)\end{array}$ & 0.845 \\
\hline
\end{tabular}

Las causas de enfermedad renal fueron: glomerulonefritis (2), diabetes mellitus (2), nefroangioesclerosis (2) y otros motivos (1).

La tabla 2 muestra los valores de las pruebas funcionales de los pacientes del grupo intradiálisis y grupo domiciliario antes y después de la realización del programa. No se observaron diferencias significativas en ninguna de las pruebas de capacidad funcional. Sin embargo podemos observar que el grupo intradiálisis mejora en alguna pruebas de capacidad funcional como son el equilibrio monopodal (mediana pre 5.3 segundos, mediana post 10.9 segundos), STS 60 (mediana pre 19.5 repeticiones, mediana post 22.5 repeticiones). Se puede observar también que el valor mínimo mejora también en algunas pruebas funcionales como son en. el valor mínimo en la Dinamometría de la mano derecha que pasó de $20.5 \mathrm{~kg}$ a $25 \mathrm{~kg}$. Y por último en la prueba del 6MWT el valor mínimo de los metros recorridos fue 366 y al terminar el programa de ejercicio aumentó a 414 metros.

En el grupo domiciliario podemos observar que el valor mínimo de la prueba SPPB pasa de 8 puntos a 11 puntos. En la prueba STS-60 hay una disminución del número de repeticiones en los valores máximos (pre max 41; post max 25). En la dinamometría de mano izquierda podemos observar un ligero aumento de fuerza. Y por último en la prueba de Triceps Sural de la pierna izquierda se observa un aumento de la mediana pre (13 repeticiones) con respecto de la mediana post (25 repeticiones).

En la Tabla 3 se describen los resultados de los cuestionarios de nivel de actividad que los pacientes tuvieron que rellenar antes y después del programa de ejercicios. Se puede observar que únicamente en el cuestionario HAP hay diferencias significativas en el factor tiempo $(\mathrm{P}<.017)$ donde la mediana de los pacientes del GI tenían una puntuación pre de 48.5 ( $\min 44$ - $\max 53$ ) y post de 62 ( $\min 8$ - max 66); mientras en el HB obtuvieron una puntuación pre de $7(\min 2-\max 54)$ y post de 17.5 $(\min 13 \mathrm{y}-\max 86)$.

En cuanto a la adherencia a los programas de ejercicio los pacientes del GI cumplieron el $92.7 \%$ del total de las sesiones, mientras que el grupo HB cumplió el $68.7 \%$ del total de las sesiones.

\section{Discusión y conclusión}

Los resultados de este estudio indican que el nivel de actividad con un programa de ejercicio de 4 meses aumenta. Se ha observado una diferencia en cuanto el nivel de actividad física en el cuestionario HAP en el factor tiempo en la puntuación de actividad ajustada (ASS), aumentando en ambos grupos (en el GI la mediana del HAP AAS pre fue 48.5 puntos con un mínimo de 44 y un máximo de 53 puntos y el post la mediana fue de 62 puntos con un mínimo de 58 y un máximo de 66; mientras que en el grupo HB la mediana pre del HAP AAS fue de 7 con un mínimo de 2 y un máximo de 54 puntos y la post de 17.5 puntos con un mínimo de 13 y un máximo de 86). Este cuestionario se ha relacionado con el nivel de actividad de las personas con IRC y proporciona una estimación más estable de las actividades diarias de la persona. Esto significa que pasan de ser considerados personas inactivas a persona moderada- 
Tabla 2. Pruebas de capacidad funcional.

\begin{tabular}{|c|c|c|c|c|c|c|c|}
\hline VARIABLES & Hemodiálisis & Domicilio & $\mathbf{n}$ & $\begin{array}{l}\text { F tiempo } \\
\text { *grupo }\end{array}$ & $\mathbf{p}^{\mathrm{a}}$ & F tiempo & $\mathbf{p}^{\mathbf{b}}$ \\
\hline \multicolumn{8}{|l|}{ SPPB (0-12 puntos) } \\
\hline Pre Mediana(Min-Max) & $11(10-12)$ & $10(8-12)$ & & & & & \\
\hline \multirow{2}{*}{ Post Mediana (Min-Max) } & $11,5(11-12)$ & $11(11-12)$ & & & & & \\
\hline & & & 7 & 0.660 & 0.453 & 3.895 & 0.105 \\
\hline \multicolumn{8}{|l|}{ Equilibrio Monopodal (45 segundos) } \\
\hline Pre Mediana(Min-Max) & $5.33(4.07-6.60)$ & $6.53(1-45)$ & & & & & \\
\hline \multirow[t]{2}{*}{ Post Mediana (Min-Max) } & $10.85(1.96-19.75)$ & $4.18(1.59-45)$ & & & & & \\
\hline & & & 7 & 0.688 & 0.445 & 0.575 & 0.483 \\
\hline \multicolumn{8}{|l|}{ TUG (segundos) } \\
\hline Pre Mediana (Min-Max) & $7.75(7.25-8.25)$ & 8.78(6.19-11.91) & & & & & \\
\hline \multirow[t]{2}{*}{ Post Mediana (Min-Max) } & $7.43(7.06-7.81)$ & $8.34(7.03-11.09)$ & & & & & \\
\hline & & & 7 & 0.037 & 0.855 & 0.931 & 0.379 \\
\hline \multicolumn{8}{|l|}{ STS 10 (segundos) } \\
\hline Pre Mediana (Min-Max) & $22.51(19.78-25.25)$ & $17.47(14.57-22.82)$ & & & & & \\
\hline \multirow[t]{2}{*}{ Post Mediana (Min-Max) } & $22.68(21.06-24.30)$ & $21.34(16.81-22.31)$ & & & & & \\
\hline & & & 7 & 0.307 & 0.603 & 0.454 & 0.531 \\
\hline \multicolumn{8}{|l|}{ STS 60 (repeticiones) } \\
\hline Pre Mediana (Min-Max) & $19.5(19-20)$ & $22(16-41)$ & & & & & \\
\hline \multirow[t]{2}{*}{ Post Mediana (Min-Max) } & $22.5(22-23)$ & $22(18-25)$ & & & & & \\
\hline & & & 7 & 0.574 & 0.483 & 0.001 & 0.980 \\
\hline \multicolumn{8}{|l|}{ Dinamometria mano Dcha $(\mathrm{Kg})$} \\
\hline Pre Mediana (Min-Max) & $26.25(20.5-32)$ & $34(13-40)$ & & & & & \\
\hline \multirow[t]{2}{*}{ Post Mediana (Min-Max) } & $29.5(25-34)$ & $30(16-39)$ & & & & & \\
\hline & & & 7 & 1.321 & 0.302 & 1.321 & 0.302 \\
\hline \multicolumn{8}{|l|}{ Dinamometria mano Izq $(\mathrm{Kg})$} \\
\hline Pre Mediana (Min-Max) & $27(26-28)$ & $24(10-32)$ & & & & & \\
\hline \multirow[t]{2}{*}{ Post Mediana (Min-Max) } & $27(26-28)$ & $26(14-34)$ & & & & & \\
\hline & & & 7 & 3.673 & 0.113 & 3.673 & 0.113 \\
\hline \multicolumn{8}{|l|}{ Triceps Sural Dch (repeticiones) } \\
\hline Pre Mediana (Min-Max) & $23.5(22-25)$ & $20(3-25)$ & & & & & \\
\hline \multirow[t]{2}{*}{ Post Mediana (Min-Max) } & $23.5(22-25)$ & $15(0-25)$ & & & & & \\
\hline & & & 7 & 0.006 & 0.941 & 0.006 & 0.941 \\
\hline \multicolumn{8}{|l|}{ Triceps Sural izq (repeticiones) } \\
\hline Pre Mediana (Min-Max) & $19(13-25)$ & $13(0-25)$ & & & & & \\
\hline \multirow[t]{2}{*}{ Post Mediana (Min-Max) } & $25(25-25)$ & $25(0-25)$ & & & & & \\
\hline & & & 7 & 0.220 & 0.659 & 3.516 & 0.120 \\
\hline \multicolumn{8}{|l|}{ 6MWT (metros) } \\
\hline Pre Mediana (Min-Max) & $413(366-460)$ & $387(320-525)$ & & & & & \\
\hline \multirow[t]{2}{*}{ Post Mediana (Min-Max) } & $439(414-464)$ & $380(348-522)$ & & & & & \\
\hline & & & 7 & 0.889 & 0.389 & 2.594 & 0.168 \\
\hline
\end{tabular}

mente activas según la clasificación que se da para el $\mathrm{HAP}^{8,31}$. Como se puede ver en los resultados hay una diferencia significativa en ambos grupos pero no entre grupos, por tanto, se puede realizar un programa de ejercicio domiciliario, el cuál es más económico, para aumentar la actividad de esta población. Aunque este hallazgo se debería confirmar con estudios con un número de muestra mayor.
El porcentaje de nivel de adherencia entre el grupo de ejercicio durante la hemodiálisis con respecto al grupo domiciliario vemos que el primero ha realizado mayor número de sesiones que el grupo que tenía que realizar el ejercicio en casa. Preguntando de manera subjetiva a los participantes por las barreras que habían encontrado para hacer el ejercicio, nos comentaron que al no tener a nadie con quien realizar el ejercicio les costaba 
Tabla 3. Nivel de actividad funcional.

\begin{tabular}{|c|c|c|c|c|c|c|c|}
\hline VARIABLES & Hemodiálisis & Domicilio & $\mathbf{n}$ & $\begin{array}{l}\text { F tiempo } \\
\text { * grupo }\end{array}$ & $\mathbf{p}^{\mathrm{a}}$ & F tiempo & $\mathbf{p}^{\mathrm{b}}$ \\
\hline \multicolumn{8}{|l|}{ PASE (0-100) } \\
\hline Pre Mediana (Min-Max) & $35.6(20.4-50.8)$ & $51.5(5-107.4)$ & & & & & \\
\hline \multirow{2}{*}{ Post Mediana (Min-Max) } & $73.2(47.8-98.6)$ & $64.4(58.6-128.5)$ & & & & & \\
\hline & & & 6 & 0.256 & 0.640 & 6.473 & 0.064 \\
\hline \multicolumn{8}{|l|}{ HAP MAS(puntuación) } \\
\hline Pre Mediana (Min-Max) & $67.5(66-69)$ & $48.5(48-73)$ & & & & & \\
\hline \multirow[t]{2}{*}{ Post Mediana (Min-Max) } & $77(75-79)$ & $53.5(52-90)$ & & & & & \\
\hline & & & 7 & 0.710 & 0.503 & 0.050 & 0.830 \\
\hline \multicolumn{8}{|l|}{ HAP AAS(puntuación) } \\
\hline Pre Mediana (Min-Max) & $48.5(44-53)$ & $7(2-54)$ & & & & & \\
\hline \multirow{2}{*}{ Post Mediana (Min-Max) } & $62(58-66)$ & $17.5(13-86)$ & & & & & \\
\hline & & & 7 & 0.090 & 0.777 & 12.470 & 0.017 \\
\hline
\end{tabular}

mucho más ponerse a hacerlo ya que siempre estaban ocupados con otras tareas y que no había nadie en sus casas que les insistiera. Estas barreras han sido descritas por otros artículos ${ }^{32}$. Para que hubiese una mayor adhesión al programa de ejercicio se les explicó cuáles eran los beneficios de realizar los ejercicios, lo cual también tenían descrito en el folleto que se les entregó. Se intentó acudir un día a su casa para explicarles el programa y así coincidir con algún familiar para que se involucraran, pero únicamente dos personas quisieron que acudiéramos a su domicilio. Se realizó un seguimiento donde se les preguntaba los días de diálisis si estaban haciendo el programa y se les insistía en los beneficios que obtendrían. En comparación con En el estudio de Tao et al 2015 donde se lleva a cabo también un programa de ejercicio domiciliario, las propias enfermeras realizaron una serie de estrategias de educación para que el paciente reforzara el ejercicio. Éstas se sentaban con cada paciente entre 15 y 30 minutos y les explicaban cuáles eran los beneficios de realizar el ejercicio, buscaron las posibles barreras que podían encontrar en casa e intentaban buscar una solución, llegaban a un consenso para marcarse unas metas y monitorizaban el ejercicio ${ }^{33}$. En este estudio también se hablaba tanto con el médico como con los familiares para hacer frente a las barreras. Sin embargo, los pacientes de este estudio que realizaron el programa a domicilio no querían que fuéramos a sus casas para hacer más partícipes a sus familiares. En futuros estudios se debería ampliar la muestra y se hacer más partícipes tanto los profesionales sanitarios de la propia unidad como a los propios pacientes y familiares. Se ha demostrado que el apoyo de los médicos y la gestión individualizada del programa hace que se contribuya al éxito de los programas domiciliarios ${ }^{33}$.
El principal hallazgo que podemos encontrar en este artículo es que después de realizar una comparación en cuanto a la capacidad funcional con diferentes pruebas funcionales no encontramos ninguna diferencia significativa entre ambos grupos. Esto puede ser debido al reducido tamaño de la muestra que se ha utilizado y es posible que el programa de ejercicio, tanto el del grupo intradiálisis como en el del grupo domiciliario, no fueran los más acertados ya que la mejora en cuatro meses no es significativa en ningún grupo.

En el futuro, estudios controlados aleatorios deberían tener un tamaño muestral mayor al del presente estudio, donde se prevea la pérdida de sujetos debido a la alta comorbilidad. Ello conllevaría a clarificar si sería factible realizar un programa de ejercicio domiciliario donde los participantes tuvieran una mayor adherencia al programa de ejercicio, así como si las pruebas de capacidad funcional y los cuestionarios que miden el nivel de actividad tuviesen una diferencia significativa entre los resultados antes y después de un programa de ejercicio que combine fuerza muscular y ejercicio aeróbico y el efecto que produce sobre en la calidad de vida de este tipo de población.

Por otra parte, vemos qué efecto puede producir que un fisioterapeuta esté en una unidad de diálisis para que haya una mayor adherencia al programa de ejercicio y una mayor predisposición por parte del paciente a realizar el ejercicio. Aún así, habría que incentivar la participación de aquellos pacientes de mayor edad, dada la baja predisposición observada a realizar ejercicio.

A pesar de estar demostrado que el ejercicio durante la hemodiálisis tiene beneficios para los pacientes con IRC 
en su calidad de vida relacionada con la salud, las unidades de diálisis no tienen personal cualificado que monitorice programas de ejercicio en esta población. Es por ello que se puso en marcha este estudio, con el objetivo de ver el efecto de realizar ejercicio en el domicilio. Que tengamos constancia, es la primera vez que se aplica un programa de ejercicio en casa para pacientes con IRC en España. El incremento de la actividad física de los enfermos que están obligados tener este tipo de tratamiento y que supone un número elevado de horas en la posición de sedestación o en decúbito supino, puede ser un medio para así disminuir el deterioro musculo-esquelético en estos pacientes.

Podemos concluir que tanto el ejercicio durante la hemodiálisis como el ejercicio domiciliario resultan en un aumento del nivel de actividad física de los pacientes con insuficiencia renal crónica en tratamiento de hemodiálisis. Es por ello que consideremos necesario modificar factores, tanto en el personal sanitario que atiende a estos pacientes, como en los propios pacientes, para conseguir mayor adherencia a los programa de ejercicio.

\section{Agradecimientos}

Agradecer al Servicio de Nefrología del Hospital Virgen del Consuelo de Valencia su hospitalidad y colaboración para poder llevar a cabo este estudio, así como a todo el Departamento de Fisioterapia de la Universidad CEU Cardenal Herrera.

Recibido: 10 octubre 2015

Revisado:23 octubre 2015

Modificado: 2 diciembre 2015

Aceptado:12 diciembre 2015

\section{Bibliografía}

1. Deligiannis A, Kouidi E, Tassoulas E, Gigis P, Tourkantonis A, Coats A. Cardiac effects of exercise rehabilitation in hemodialysis patients. Int $\mathrm{J}$ Cardiol 1999 Aug 31;70(3):253-266.

2. Goldberg AP, Geltman EM, Gavin JR,3rd, Carney RM, Hagberg JM, Delmez JA, et al. Exercise training reduces coronary risk and effectively rehabilitates hemodialysis patients. Nephron 1986;42(4):311-316.
3. Johansen KL, Shubert T, Doyle J, Soher B, Sakkas GK, Kent-Braun JA. Muscle atrophy in patients receiving hemodialysis: effects on muscle strength, muscle quality, and physical function. Kidney Int 2003 Jan;63(1):291-297.

4. Johansen KL, Doyle J, Sakkas GK, Kent-Braun JA. Neural and metabolic mechanisms of excessive muscle fatigue in maintenance hemodialysis patients. Am J Physiol Regul Integr Comp Physiol 2005 Sep;289(3):R805-13.

5. Kouidi E, Albani M, Natsis K, Megalopoulos A, Gigis P, Guiba-Tziampiri 0, et al. The effects of exercise training on muscle atrophy in haemodialysis patients. Nephrol Dial Transplant 1998 Mar;13(3):685-699.

6. Mitnitski A, Bao L, Skoog I, Rockwood K. A crossnational study of transitions in deficit counts in two birth cohorts: implications for modeling ageing. Exp Gerontol 2007 Mar;42(3):241-246.

7. Mitnitski A, Fallah N, Rockwood MR, Rockwood K. Transitions in cognitive status in relation to frailty in older adults: a comparison of three frailty measures. J Nutr Health Aging 2011 Dec;15(10):863-867.

8. Álvarez-Ude, F., Fernández-Reyes, M.J., Vázquez, A., Mon, C., Sánchez, R. \& Rebollo, P. Physical symptoms and emotional disorders in patient on a periodic hemodialysis program. Nefrología: publicación oficial de la Sociedad Española Nefrología, 2001; 21(2):191-199.

9. Johansen $K L$, Painter $P$, Kent-Braun JA, Ng AV, Carey $S$, Da Silva $M$, et al. Validation of questionnaires to estimate physical activity and functioning in end-stage renal disease. Kidney Int 2001 Mar;59(3):1121-1127.

10. Heiwe S, Tollback A, Clyne N. Twelve weeks of exercise training increases muscle function and walking capacity in elderly predialysis patients and healthy subjects. Nephron 2001 May;88(1):48-56.

11. Avesani CM, Trolonge S, Deleaval P, Baria F, Mafra $D$, Faxen-Irving $G$, et al. Physical activity and energy expenditure in haemodialysis patients: an international survey. Nephrol Dial Transplant 2012 Jun; 27(6):2430-2434.

12. Painter P. Physical functioning in end-stage renal disease patients: update 2005. Hemodial Int 2005 Jul;9(3):218-235. 
13. Johansen $\mathrm{KL}$. Exercise in the end-stage renal disease population. J Am Soc Nephrol 2007 Jun;18(6):1845-1854.

14. Peng YS, Chiang CK, Hung $\mathrm{KY}$, Chang $\mathrm{CH}$, Lin $C Y$, Yang CS, et al. Are both psychological and physical dimensions in health-related quality of life associated with mortality in hemodialysis patients: a 7-year Taiwan cohort study. Blood Purif 2010;30(2):98-105.

15. Painter $P$, Messer-Rehak D, Hanson P, Zimmerman SW, Glass NR. Exercise capacity in hemodialysis, CAPD, and renal transplant patients. Nephron 1986;42(1):47-51.

16. Konstantinidou E, Koukouvou G, Kouidi E, Deligiannis A, Tourkantonis A. Exercise training in patients with end-stage renal disease on hemodialysis: comparison of three rehabilitation programs. J Rehabil Med 2002 Jan;34(1):40-45.

17. Deligiannis A. Exercise rehabilitation and skeletal muscle benefits in hemodialysis patients. Clin Nephrol 2004 May;61 Suppl 1:S46-50.

18. Painter, P., Clark, L., Olausson,J.Physical function and physical activity assessment and promotion in the hemodialysis clinic:a qualitative study. Am.J.Kidney Dis. 2014 Jan; 64(3),425-433.

19. Ostir GV, Ottenbacher KJ, Fried LP, Guralnik JM. The Effect of Depressive Symptoms on the Association Between Functional Status and Social Participation. Soc Indic Res 2007 Jan;80(2):379392.

20. Hurvitz, E. A., Richardson, J. K., \& Werner, R. A. Unipodal stance testing in the assessment of peripheral neuropathy. Archives of Physical Medicine and Rehabilitation. 2001; 82(2), 198204.

21. Podsiadlo D, Richardson S: The time "up \& go": a test of basic functional mobility for frail elderly persons. J Am Geriatr Soc 1991, 39(2):142-148. 36.

22. Haweewannakij T, Wilaichit $S$, Chuchot $R$, Yuenyong $Y$, Saengsuwan J, Siritaratiwat $W$, et al. Reference values of physical performance in Thai elderly people who are functioning well and dwelling in the community. Phys Ther 2013 0ct;93(10):1312-1320.
23. Silva LF, Matos CM, Lopes GB, Martins MT, Martins MS, Arias LU, et al. Handgrip strength as a simple indicator of possible malnutrition and inflammation in men and women on maintenance hemodialysis. $J$ Ren Nutr 2011 May;21(3):235-245.

24. Leal V0, Mafra D, Fouque D, Anjos LA. Use of handgrip strength in the assessment of the muscle function of chronic kidney disease patients on dialysis: a systematic review. Nephrol Dial Transplant 2011 Apr;26(4):1354-1360.

25. Csuka M, McCarty DJ. Simple method for measurement of lower extremity muscle strength. Am J Med. 1985;78:77-81.

26. Svantesson U, Osterberg U, Thomee R, Grimby G. Muscle fatigue in a standing heel-rise test. Scand $J$ Rehabil Med. 1998; 30:67-72.

27. Portney LG, Watkins MP. Foundations of Clinical Research: Applications to Practice. 2nd ed. Upper Saddle River, NJ: Prentice Hall Health; 2000.

28. Sekir U, Yildiz Y, Hazneci B, et al. Reliability of a functional test battery evaluating functionality, proprioception, and strength in recreational athletes with functional ankle instability. Eur J Phys Rehabil Med. 2008;44:407-415.

29. ATS Committee on Proficiency Standards for Clinical Pulmonary Function Laboratories. ATS statement: guidelines for the six minute walk test. Am J Respir Crit Care Med. 2002;166:111-117.

30. Fix, A. \& Daughton, D., Human Activity Profile (HAP) Manaul. Psychological Assessment Resources Inc., Odessa 1986.

31. Overend T, Anderson C, Sawant A, Perryman $B$, Locking-Cusolito $H$. Relative and absolute reliability of physical function measures in people with end-stage renal disease. Physiother Can 2010 Spring;62(2):122-128.

32. Heiwe S, Tollin H. Patients' perspectives on the implementation of intra-dialytic cycling--a phenomenographic study. Implement Sci 2012 Jul $25 ; 7: 68$.

33. Tao X, Chow SK, Wong FK. A nurse-led case management program on home exercise training for hemodialysis patients: A randomized controlled trial. Int J Nurs Stud 2015 Jun;52(6):1029-1041. 